\title{
Mental health technology assessment: practice based research to support evidence-based practice
}

\section{Evidence-based health care and health technology assessment}

Evidence-based health care needs evidence, or more specifically, high quality research based evidence to answer the specific questions generated by clinicians and their patients in making diagnostic and therapeutic decisions. The problem is that research has traditionally not been focused on these needs. Hitherto, the main drivers of the research agenda have been:

- Industry

- The enquiring minds of clinical and non-clinical scientists, supported and guided by funders (in the UK, governmental organisations, such as the Medical Research Council, and charities are important)

- Enthusiasts working in routine clinical practice (as the demands of work allow).

In the UK, the National Health Service's (NHS) research and development strategy, launched in 1991, sought "to create a knowledge-based health service in which clinical, managerial and policy decisions are based on sound information about research findings and scientific developments." ${ }^{1}$ The health technology assessment (HTA) programme was established as part of this and has the aim:

"To ensure that high quality research information on the costs, effectiveness and broader impact of health technologies is produced in the most efficient way for those who use, manage and work in the NHS." ${ }^{2}$

The term "health technologies," although it may conjure up images of gleaming complex machinery attended upon by white coated technocrats, is used throughout the world in a broad way. In common with others, the HTA programme defines health technologies as:

"all methods used by health professionals to promote health, prevent and treat disease and improve rehabilitation and care."

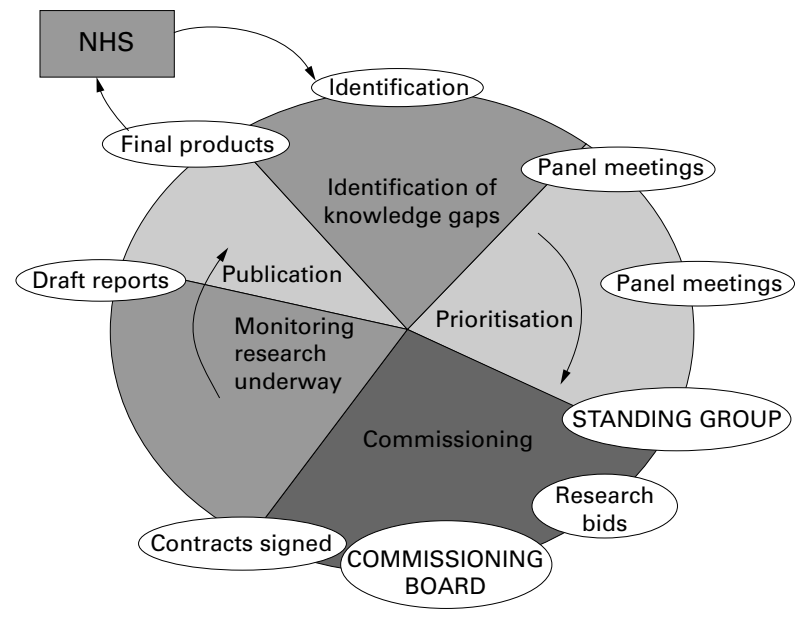

Figure 1 The HTA cycle.
All mental health interventions (drugs, devices, procedures, and settings) therefore fall well within the remit of the programme. HTA is the evaluation of the costs, effectiveness, and broader impact of health technologies. The endpoints of evaluation should be patient centred outcomes and estimates of the cost effectiveness of technologies. HTA is therefore pragmatic. It aims to inform clinical and health policy decision making rather than to establish efficacy.

\section{The NHS HTA programme and the international HTA scene}

The HTA programme is ongoing, beginning a cycle each year of identifying and prioritising needs for research, commissioning, and monitoring research projects and communicating the results of research back to the NHS (fig 1). The National Coordinating Centre for Health Technology Assessment (NCCHTA) was established in 1996 at the Universities of Southampton and York to support all aspects of the HTA programme.

The NHS HTA programme is part of a growing HTA worldwide community, with the umbrella organisations of the International Society for Technology Assessment in Health Care $^{3}$ and the International Network of Agencies for Health Technology Assessment ${ }^{4}$ providing for the development of methods, exchange of ideas, and promotion of joint working. The NHS programme has one of the largest portfolios of HTA work and is unusual in 2 ways: firstly, the extent to which it seeks to be driven by the expressed needs of the health service; and secondly, by the inclusion of primary and methodological research as well as systematic reviews.

Although the nature and relationships of HTA agencies to national and local policy making (including the regulation of technologies such as drugs) varies considerably internationally, the theme they all share is research as a support to decision making. But important differences exist. In the US, there is a broader range of types of HTA agency, from the Agency for Health Care Policy and Research (AHCPR) Center for Practice and Technology Assessment, ${ }^{5}$ through for-profit independent agencies to inhouse agencies within healthcare provider organisations. In Canada, Spain, and to some extent the UK, there are both regional and national organisations, making coordination and communication between agencies important. In the UK this is achieved through InterDEC, ${ }^{6}$ which brings together regional HTA departments doing secondary research and links closely to the national programme.

In New Zealand, the national HTA agency also acts as the clearing house for health outcomes and offers a range of products based around increasing detail of literature review and involvement of experts in appraisal, interpretation, and recommendation on the basis of the evidence. ${ }^{7}$ This flexibility emphasises the way HTA seeks to respond to the needs of decision makers, where there may be a need to trade 
- Ambulatory care in acute psychiatry (Netherlands)

- The appropriateness of psychotropic treatment (antidepressants and anxiolytics) in primary care (Catalonia)

- Assessment of a routine telepsychiatry service (AHCPR, USA)

- Attention deficit hyperactivity disorder (the Netherlands)

- Cognitive behavioural therapy for patients with chronic fatigue syndrome (the Netherlands)

- Cost effectiveness of different organisational models for treatment of people with severe and persistent mental health problems (Quebec)

- Counselling of patients with chronic depression and anxiety (UK NCCHTA)

- Depression (CG) (Denmark)

- Drug addiction: medical interventions (the Netherlands)

- Effectiveness of counselling, cognitive behavioural therapy, and general practitioner care for depression in general practice (UK NCCHTA)

- Effectiveness of day care for people with severe mental disorders (UK NCCHTA)

- Electroconvulsotherapy (Quebec)

- Health status measures for people with cognitive impairment (including learning disability) (UK NCCHTA)

- Interdisciplinary assessment of dementia (Denmark)

- Long term psychotherapy (the Netherlands)

- Methylphenidate (Ritalin) for attention deficit disorder (summary) (Canada)

- New drugs for Alzheimer's disease (Canada)

- New drugs for psychosis (Sweden)

- New neuroleptics in schizophrenia (modelling and review) (UK NCCHTA)

- Organo-psychosyndrome: limits for exposure to solvents (the Netherlands)

- Pharmacotherapy and other treatment options for Alzheimer's disease (the Netherlands)

- Psychological treatment in the regulation of long term hypnotic drug use (UK NCCHTA)

- Risperidone $v$ olanzapine in schizophrenia (Canada)

- Schizophrenia: screening, early diagnosis, and treatment (the Netherlands)

- Seclusion and restraint as a method of treatment for people with serious mental illnesses (Finland)

- A systematic review of randomised and nonrandomised intervention studies to examine which of the brief psychological treatments used in primary care lead to improved outcomes (UK NCCHTA)

- Systematic reviews of (i) comparative studies of depot neuroleptic drugs, and (ii) studies of oral $v$ depot neuroleptic agents for patients with schizophrenia: clinical, social, and economic outcomes (UK NCCHTA)

- Treatment of alcohol and drug related problems (Sweden)

- Treatment of depression (Sweden)

- Treatment of anxiety (Sweden)

- Therapy with neuroleptics (Sweden)

- Treatment of drug addicts in detention (the Netherlands)

Box 1 Some mental health projects underway in the International Network of Agencies for Health Technology Assessment ${ }^{15}$

timeliness against exhaustive methodology-a familiar situation to all of us who have tried to make daily practice evidence based.

Box 1 shows considerable overlap in international HTA activity, but we believe this is to be expected. The reason is that the context for the use of health technologies (especially people based technologies, such as community mental health teams) is crucial to the assessment of their value in practice. An important methodological question for evidence-based health care and HTA is "how much can we use the results of research in different places and at different times?" This is being addressed by several projects within the UK HTA programme. ${ }^{89}$

\section{The HTA cycle}

In the UK, NCCHTA identifies information gaps that matter to decision makers at all levels in the NHS. This is done by various means:

- Postal consultation, for example with the Royal College of Psychiatrists

- Focus group discussions, for example in 1998 with a group from the Mental Health Foundation

- Suggestions made through the NCCHTA website ${ }^{10}$

- Systematic screening of systematic reviews from the Cochrane Collaboration and the HTA programme itself, for example this generated the priority in 1998 for a trial of brief psychological interventions following deliberate self harm

- Reconsideration of subjects which were given some priority but not eventually supported for funding, and horizon scanning, which identified in 1995 the need for evaluation of new drugs for Alzheimer's disease.

This last process involves trying to identify which technologies are likely to have the highest impact on health services within the next few years so that research can be commissioned with sufficient lead time to make findings available when the need for them is greatest. Similar early warning systems exist in several other countries, notably the Netherlands, and have been the subject of a forthcoming report from the HTA programme. ${ }^{11}$

Without meaningful input from these sources, the HTA programme cannot help but fail in meeting its aim of serving the needs of the NHS. Happily, there is no shortage of ideas for assessment; this year we have received around 1500 suggestions. However, many of these are not sufficiently clear to proceed easily through the prioritisation process. No effort spent clarifying a question is ever wasted, either in informing our own practice of evidence-based health care, focusing our own research, or specifying the research priorities of others such as the HTA programme.

The many suggestions that are fed into the HTA programme each year are prioritised by 5 expert clinical panels and the methodology panel, which considers how methods for HTA can be improved. A strength of the programme is a pragmatic, service oriented arrangement of the clinical panels. Mental health, like other important health problem areas, therefore features across the entire programme. The table shows the panels and examples of mental health topics that they have prioritised in the past.

The Standing Group for Health Technology finalises the priorities suggested by the expert advisory panels and the 40 or so highest priorities become the subjects of calls for proposals from the research community. The HTA Commissioning Board considers up to 500 outline proposals for research projects in a 2 stage process peer review of more detailed full proposals. Each year the programme spends about $£ 6 m$ of the $£ 420 \mathrm{~m}$ NHS research and development budget, centrally levied from healthcare commissioners.

NCCHTA monitors over 190 projects that are underway to ensure that unforeseen problems are handled swiftly and that researchers do not "drift" away from the original question asked. This is an intensive and near unique aspect of 


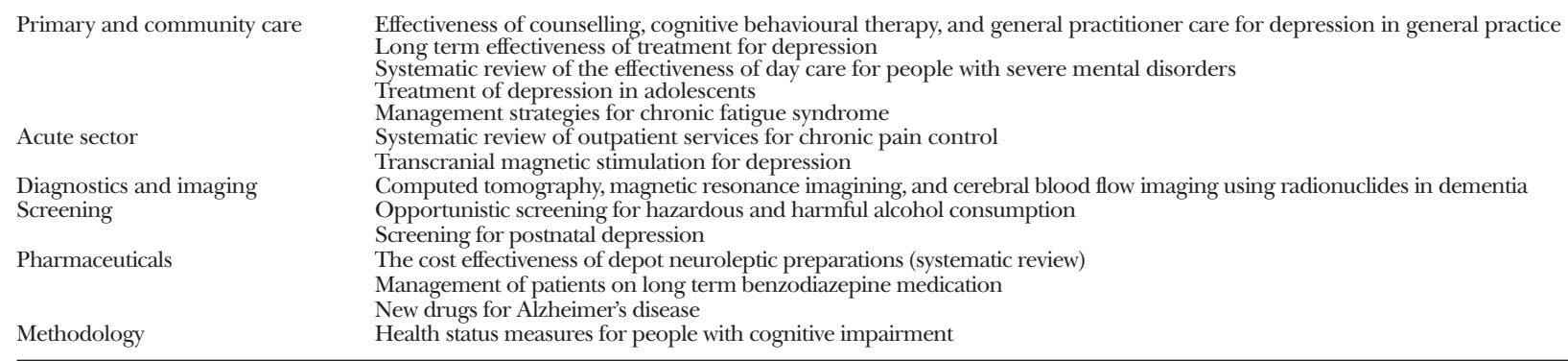

As users of research you can:

- Submit suggestions via our web site or (in the UK) through your local NHS research and development directorate

- Obtain and use reports of HTA research from our web site, by asking NCCHTA or through your own library

As doers of research you can:

- Watch our web site for news of the next call for proposals or

- Read the $B M J$ and other journals where the call is advertised

Box 2 What's in it for you?

the programme and emphasises the fact that this is the NHS's research, and not simply a vehicle for the curiosity of academics!

Finally, NCCHTA receives the findings of research projects. The need now is to "close the loop" with the NHS and to make these available. In harmony with the ethos of evidence-based, problem led practice, we have avoided bombarding the unsuspecting and unwilling NHS community with unsolicited information arising from HTA research. Instead we have tried to ensure that whenever practitioners ask a question that can be answered by an HTA report, that they will find it by using the most popular information sources available. An important UK initiative to make high quality health information more widely available, into which the HTA programme will link, is the National Electronic Library for Health. ${ }^{12}$

A changing feature of the HTA programme (in which other countries may have the lead) is the development of more explicit links to national policy development. The National Screening Committee, for instance, considers all HTA screening reports. The establishment of the national institute for Clinical Excellence and national service frameworks, ${ }^{13}$ two influential changes in the UK health service designed to enhance the quality of care, will provide important opportunities for the findings of HTA research to influence practice.

Full reports of HTA funded projects are edited and published in a monograph series Health Technology Assessment which is listed in Medline and Embase. Systematic reviews are listed in the Database of Reviews of Effectiveness (DARE) and, where relevant, in the Cochrane Database of Systematic Reviews, both part of the Cochrane Library. Copies of reports are available free of charge to those working in the UK public sector and can be ordered (or downloaded) over the world wide web. ${ }^{14}$ Please visit it at http://www.soton.ac.uk/ hta and tell us how to improve!

KEN STEIN, MB ChB, MSc, MRCGP, MFPHM Consultant in Public Health Medicine, North and East Devon Health Authority, UK RUAIRIDH MILNE, MB BS, MSc, FFPHM Scientific Director, National Coordinating Centre for Health Technology Assessment, University of Southamptom, UK

1 Peckham M. Research for health: a research and developments for the NHS. London: HMSO, 1991.

2 Department of Health. The annual report of the NHS health technology assessment programme. London: $\mathrm{DoH}, 1998$.

h http:/ www.hardboiledegg.com/istahc//index/htm

5 http://www.ahcpr.gov/

$6 \mathrm{http}: / /$ www.soton.ac.uk/ interdec/

$7 \mathrm{http://nzhta.chmeds.ac.nz/}$

8 Project 98/22: How stable are the conclusions of cost effectiveness analyses in place and time? See list of projects on the web at http://www.sonton.sc.uk/ hta/research.htm

9 Project 93/51/02: Assessing and increasing the generalisability of RCTs. View on the web at http://www.soton.ac.uk/ hta/projects/935102.htm

10 http://www.soton.ac.uk/ hta/form.htm

1 Robert G, Stevens A, Gabbay J. Early warning systems for identifying new health care technologies. Health Technology Assessment 1999 (in press)

12 http://www.nelh.nhs.uk

13 Department of Health. A first class service: quality in the new NHS. London: DoH, 1998.

14 http://www.soton.ac.uk/ hta/htapubs.htm

15 More details can be found via http://nzhta.chmeds.ac.nz/inahta/ inahta.htm 